\title{
Interaction between Autonomic Regulation, Adiposity Indexes and Metabolic Profile in Children and Adolescents with Overweight and Obesity
}

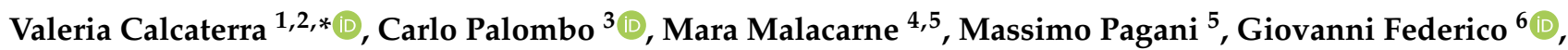 \\ Michaela Kozakova ${ }^{3}$, Gianvincenzo Zuccotti ${ }^{1,7}$ (D) and Daniela Lucini ${ }^{4,5, *}$ \\ 1 Pediatric Department, “V. Buzzi” Children's Hospital, 20154 Milan, Italy; gianvincenzo.zuccotti@unimi.it \\ 2 Pediatric and Adolescent Unit, Department of Internal Medicine, University of Pavia, 27100 Pavia, Italy \\ 3 Department of Surgical, Medical, Molecular Pathology and Critical Area Medicine, University of Pisa, \\ 56126 Pisa, Italy; carlo.palombo@unipi.it (C.P.); m.kozakova@int.med.unipi.it (M.K.) \\ 4 BIOMETRA Department, University of Milan, 20133 Milan, Italy; mara.malacarne@unimi.it \\ 5 Exercise Medicine Unit, Istituto Auxologico Italiano, 20133 Milan, Italy; massimo.paganiz@gmail.com \\ 6 Pediatric Endocrinology and Diabetes Unit, Department of Clinical and Experimental Medicine, University of \\ Pisa, 56126 Pisa, Italy; Giovanni.Federico@med.unipi.it \\ 7 Department of Biomedical and Clinical Science “L. Sacco”, University of Milan, 20157 Milan, Italy \\ * Correspondence: valeria.calcaterra@unipv.it (V.C.); daniela.lucini@unimi.it (D.L.)
}

check for updates

Citation: Calcaterra, V.; Palombo, C.; Malacarne, M.; Pagani, M.;

Federico, G.; Kozakova, M.;

Zuccotti, G.; Lucini, D. Interaction

between Autonomic Regulation,

Adiposity Indexes and Metabolic

Profile in Children and Adolescents

with Overweight and Obesity.

Children 2021, 8, 686. https://

doi.org/10.3390/children8080686

Academic Editor: Stella Stabouli

Received: 3 June 2021

Accepted: 4 August 2021

Published: 9 August 2021

Publisher's Note: MDPI stays neutra with regard to jurisdictional claims in published maps and institutional affiliations.

Copyright: (C) 2021 by the authors. Licensee MDPI, Basel, Switzerland. This article is an open access article distributed under the terms and conditions of the Creative Commons Attribution (CC BY) license (https:/ / creativecommons.org/licenses/by/ $4.0 /)$
Abstract: Early obesity predicts initial modifications in cardiac and vascular autonomic regulation. The aim of this study was to assess the possible interaction between non-invasive measures of autonomic cardiovascular control and peripheral endothelium regulation in children with overweight and obesity. We involved 114 young subjects $(77 \mathrm{M} / 37 \mathrm{~F}, 12.7 \pm 2.2$ years) with normal weight (NW, $n=46)$ to overweight or obesity $(\mathrm{OB}, n=68)$. Multivariate statistical techniques utilizing a collection of modern indices of autonomic regulation, adiposity indexes and metabolic profile were employed. Resting values show substantial equivalence of data. Conversely, blood pressure variance is greater in NW/OB groups. The correlation matrix between major autonomic and metabolic/hemodynamic variables shows a clustered significant correlation between homogeneous indices. A significant correlation between metabolic indices and endothelial and autonomic control, mostly in its vascular end, was recorded. Particularly, the alpha index is significantly correlated with triglycerides $(r=-0.261)$ and endothelial indices (RHI, $r=0.276)$. Children with obesity show a link between indices of autonomic and endothelial function, fat distribution and metabolic profile. The optimization of autonomic control, for instance by exercise/nutrition interventions, could potentially prevent/delay the occurrence of structural vascular damage leading to reduced cardiovascular health.

Keywords: pediatric obesity; autonomic regulation; adiposity index; metabolic; children; adolescents; overweight; peripheral endothelium

\section{Introduction}

Pediatric obesity remains an important health problem. The prevalence of obesity increased three times from 1975 to 2016 [1]; overweight or obesity were recorded in more than 40 million children aged under the age of 5 years and 340 million children and adolescents aged 5-19 years [2]. In Italy, 17\% and 9.3\% of school-aged children were overweight or obese [3].

Body-fat excess and distribution play critical roles for health risk [4]. Body mass index (BMI), waist circumference (WC) and/or waist-to-height ratio (WHtR) are the most widely used indices in clinical practice. Even though these parameters present serious limitations, particularly in children, because they do not accurately discriminate between lean and fat mass, they are considered good markers of metabolic and cardiovascular comorbidities [5,6]. Recently, new indexes of obesity, such as triponderal mass index (TMI) [7] and non-linear equations to estimate the fat mass [8], have been proposed as 
markers of cardiovascular risk. Additionally, ultrasonography may be used as a reliable method to measure subcutaneous and visceral fat for predicting health risk [9] related to obesity.

Obesity early in life predisposes individuals to later cardiovascular and metabolic alterations [10]. In particular, early obesity strongly predicts initial modifications in cardiac and vascular autonomic regulation [10]. Importantly, increased peripheral sympathetic activation and reduced cardiac vagal modulation, possibly leading to arterial hypertension, were reported in children with overweight and obesity [11]. The sympathetic activity might play a role in modulating the elastic properties of central $[12,13]$ and peripheral arteries.

Although autonomic abnormalities are usually treated as an organic irreversible disorder, it was previously documented that autonomic abnormalities may be in a large part functional in children with overweight and obesity [12,13] and the optimization of body weight by following healthy diet and physical activity programs could be considered a crucial strategy in their prevention and treatment [14-18].

The aim of this study was to assess the possible interaction between non-invasive measures of autonomic cardiovascular control and peripheral endothelium regulation in children with overweight and obesity. To this end, we employed multivariate statistical techniques utilizing a collection of modern indices of autonomic regulation, adiposity indexes and metabolic profile $[19,20]$.

\section{Materials and Methods}

\subsection{Selection of the Patients}

Given the exploratory nature of the investigation, we designed a retrospective study employing multivariate statistical techniques utilizing a collection of modern numerical and graphical tools considering the interplay between autonomic regulation, adiposity outline and metabolic profile $[19,20]$.

We considered 114 young subjects $(77 \mathrm{M} / 37 \mathrm{~F}$, mean overall age: $12.7 \pm 2.2$ years) with normal weight $(n=46)$ to overweight or obesity $(n=68)$, consecutively referred as outpatients to the Pediatric Endocrinology and Diabetes Unit, Department of Clinical and Experimental Medicine, University of Pisa, Italy, for evaluation of metabolic status, between 1 February 2016 and 10 November 2016. Primary obesity, females and males, and a chronological age between 8 and 18 years were inclusion criteria. The presence of known secondary obesity conditions, use of any drugs, and concomitant chronic or acute illnesses were considered exclusion criteria.

In all patients, anthropometric data, metabolic evaluation, peripheral endotheliumdependent vasodilator capacity, ultrasound measurement of subcutaneous and visceral fat and autonomic evaluation were recorded.

\subsection{Anthropometric Data}

Height and weight were recorded in the morning, between 08:00 and 10:00 a.m. Standing height was measured using a Harpenden stadiometer (Holtain Ltd., Crosswell, UK) with a fixed vertical backboard and an adjustable head piece. The measurement was taken on the subject in an upright position, without shoes, with their heels together and toes apart, hands at sides, aligning the head in the Frankfort horizontal plane [21]. Weight was quantified with participants not wearing shoes and in light clothing, standing upright in the center of the scale platform (Seca, Hamburg, Germany) facing the recorder, hands at sides, and looking straight ahead [21]. Two measurements were taken for each parameter, and a third was obtained if a discrepancy was noted between the initial measurements for weight $(>500 \mathrm{~g})$ and height $(>0.5 \mathrm{~cm})$.

BMI as well as BMI z-scores were calculated using World Health Organization (WHO) reference values [22] to obtain worldwide valid results.

The study subjects were divided into two groups: normal weight group if BMI z-score was $<1$ and overweight/obese group if BMI z-score $\geq 1$ [22]. 
In addition to BMI, BMI z-score and WC adiposity indexes, including WHtR, TMI, and a non-linear equation to estimate the fat mass were calculated as follows:

- $\quad \mathrm{WHtR}=\mathrm{WC} / \mathrm{Ht}$;

- $\quad$ TMI = weight $(\mathrm{kg}) /$ height $(\mathrm{m})^{3}[23]$;

- $\quad$ Fat Mass $=$ weight $-\exp \left(0.3073 \times\right.$ height $^{2}-10.0155 \times$ weight $^{-1}+0.004571 \times$ weight $-0.9180 \times \ln ($ age $)+0.6488 \times$ age $^{0.5}+0.04723 \times$ male +2.8055$)$ [24] (exp = exponential function, $\ln =$ natural logarithmic transformation, male $=1$, female $=0$ ).

\subsection{Metabolic Evaluation}

Blood samples were collected by venipuncture, in the morning after overnight fasting [25]. As insulin resistance (IR) surrogates we considered the following:

HOMA-IR calculated as insulin resistance $=($ insulin $\times$ glucose $) / 22.5$ [26];

TyG-index calculated as $\ln [$ fasting triglycerides $(\mathrm{mg} / \mathrm{dL}) \times$ fasting plasma glucose $(\mathrm{mg} / \mathrm{dL}) / 2]$ [27]

\subsection{Endothelium-Dependent Vasodilation: RHI}

Peripheral endothelium-dependent vasodilator capacity was estimated assessing reactive hyperemia index (RHI), a measure for endothelial function, by means of an EndoPAT 2000 device (Itamar Medical Ltd., Cesarea, Israel) as previously described [28,29]. RHI was calculated on peripheral artery tonometry (PAT) signal changes with a proprietary computerized algorithm. Finger probes were used to assess arterial pulse wave amplitudes at the fingertips.

Subjects, after overnight fasting, were in a supine position for at least $20 \mathrm{~min}$ before the test, in a quiet room, with comfortable lights and temperature. The Endo-PAT pneumatic probes were placed on both index fingers to register arterial pulse wave amplitudes, i.e., the difference between the highest and lowest points of a pulse wave. The subjects were requested to remain as still as possible during the whole measurement phase. Each recording comprised $5 \mathrm{~min}$ baseline testing, $5 \mathrm{~min}$ occlusion testing, and $5 \mathrm{~min}$ postocclusion testing (hyperemic period).

Occlusion of the brachial artery was performed on the non-dominant upper arm by using a manometer cuff inflated to supra-systolic pressure (at least $40 \mathrm{mmHg}$ above the systolic BP). The computerized algorithm automatically calculated the RHI; that is, the ratio of the mean pulse wave amplitude between 90 and $150 \mathrm{~s}$ after deflation divided by a pre-occlusion period value during $210 \mathrm{~s}$ before occlusion, divided by the same ratio for the control arm to correct for changes in systemic vascular tone.

\subsection{Ultrasound}

A standard ultrasound system (MyLab 70, Esaote, Genova, Italy) equipped with a 3.5 MHz phased-array probe was used to obtained cardiac images. Conventional B-mode, M-mode and Doppler echocardiography were used for the assessment of LV mass and other measures (not employed in the present study). Ultrasonography for evaluation of subcutaneous and visceral fat was performed in subjects placed in a supine position. Maximum subcutaneous abdominal fat thickness was measured $5 \mathrm{~cm}$ above the umbilicus, as the distance between skin-fat and fat-linea alba interfaces [30]. Visceral fat was measured one $\mathrm{cm}$ above the umbilicus as the distance between the internal face of retro-abdominal muscle and the anterior wall of the aorta [31].

\subsection{Autonomic Evaluation}

On the day of the study, all subjects arrived at the laboratory about $2 \mathrm{~h}$ after a light breakfast, avoiding caffeinated beverages and heavy physical exercise in the preceding $24 \mathrm{~h}$. Recordings were always performed between 10:00 AM and 12:00 PM to account for circadian variations. After a preliminary $10-\mathrm{min}$ rest period in a supine position, allowing for stabilization, arterial pressure (AP) waveforms, ECG, and respiratory activity were 
continuously recorded over a 10-min baseline and a subsequent 7-min period of active standing, and then stored on a PC.

As described previously $[19,20]$, from the simultaneous autoregressive spectral analysis of the RR interval and SAP variability, a series of indices indirectly reflecting autonomic cardiovascular modulation are derived. The three major oscillatory components extracted from the continuous RR segment with an ad hoc SW tool are presented as absolute (in time) and normalized (dimensionless, pure numbers) units. The component around $0.25 \mathrm{~Hz}$ and strongly coherent with the major respiratory oscillation $(p<0.001)$ is defined as high frequency (HF), a lower frequency component (around $0.1 \mathrm{~Hz}$ ) is called low frequency (LF), and the lowest frequency component (around $0.0-0.03 \mathrm{~Hz}$ ) is called very low frequency (VLF, or DC) and is disregarded in short-term recordings. Additional methodological details have been recently published [19].

The simultaneous bivariate analysis of $R R$ and systolic arterial pressure variability also permits extraction of the gain between systolic arterial pressure and coherent RR variability components [32]. The derived index (alpha) is strongly linked to RR variance and provides an approximate estimate of the SAP RR (open) loop. The combination of these indices is a strong predictor of cardiac events.

It is worth noting that in recent years, interest in new methods of analysis of heart rate variability (HRV), or the physiological underpinning of various derived indices, considered markers of the antagonistic sympathetic-parasympathetic neural balance of cardiac regulation, has increased substantially. Indeed, in the Medline database the entry "Heart Rate Variability" elicits more than 50,000 hits, suggesting that HRV is the de facto noninvasive standard for the assessment of cardiac autonomic regulation. There are continuing debates and intensive scrutiny into novel methods stimulated by the strong predictive capacity of HRV in cardiac and metabolic conditions, or on the possibility of guiding training and fostering results in athletes as well as in ambulatory subjects. In the last few years, with a data driven approach, we have tried to simplify understanding of the relationship between the multiple indices produced by computer analysis of cardiac and vascular beat-by-beat variability signals using a multivariate statistical approach. This method is based on various steps, in particular the use of dimension reduction tools, such as the exploratory factor analysis [20]. With this approach we intend to assess whether the transition from normal weight to overweight/obesity combines with a worsening in the overall autonomic nervous system (ANS) condition as captured by the set of variability indicators. In this way, for instance, we demonstrated that the majority (about $80 \%$ ) of HRV and arterial pressure variability information, which is distributed across $15-20$ variables, can be reduced to only four ("physical") domains, corresponding to amplitude of signal variations ( $\mathrm{msec}$ or $\left.\mathrm{msec}^{2}\right)$, rate of pulse (beat/min) and arterial pressure load $(\mathrm{mmHg})$, as well as dimensionless (pure number, as with normalized units) oscillations. In the case of adults with obesity, the interested domains are in particular pulse, pressure and amplitude, and not oscillations. Clearly, from these indices it is not possible to obtain measures of autonomic activity, but only indices of autonomic (dys)regulation.

A simplified approach, primarily based on exploratory factor analysis and simple correlations, was employed in the present feasibility study based on a relatively small, but homogeneous, population.

\subsection{Statistics}

Data are presented in the text, figures and tables as averages \pm SD.

Statistical evaluation comprised independent $t$-test, simple Pearson's correlations, and exploratory factor analysis (EFA). Computations were performed with a commercial statistical package (SPSS 27) considering a significance threshold of $p<0.05$ (two sided).

\section{Results}

Table 1 reports summary anthropometric, hemodynamic and metabolic data in the two groups (normal weight vs. overweight/obesity subjects; i.e., NW vs. OW/OB). There 
is no difference in height between the two groups, while OW/OB subjects show, by design, a greater weight, BMI and BMI z-score, as well as Waist and Waist/Height, as compared to NW. In addition, as expected, a significant difference between the two groups in almost all metabolic indicators was noted, with the exception of HDL, glucose and TryG.

Table 1. Anthropometric, hemodynamic and metabolic data in normal weight (NW) and overweight/obese children (OW/OB).

\begin{tabular}{|c|c|c|c|}
\hline Variables & NW & OW/OB & Significance \\
\hline Height $(\mathrm{cm})$ & $158.9 \pm 12.2$ & $157.6 \pm 11.6$ & 0.549 \\
\hline Weight (kg) & $48.9 \pm 11.3$ & $72.3 \pm 18.7$ & $<0.001$ \\
\hline $\mathrm{BMI}\left(\mathrm{kg} / \mathrm{m}^{2}\right)$ & $19.07 \pm 2.04$ & $28.8 \pm 5.51$ & $<0.001$ \\
\hline BMI z score (-) & $0.11 \pm 0.65$ & $2.75 \pm 1.09$ & $<0.001$ \\
\hline Triponderal Mass Index $\left(\mathrm{kg} / \mathrm{m}^{3}\right)$ & $12.01 \pm 0.95$ & $18.34 \pm 3.56$ & $<0.001$ \\
\hline Waist circumference $(\mathrm{cm})$ & $72.0 \pm 10.1$ & $94.2 \pm 14.9$ & $<0.001$ \\
\hline WHtR (-) & $0.41 \pm 0.14$ & $0.59 \pm 0.11$ & $<0.001$ \\
\hline Visceral fat $(\mathrm{cm})$ & $2.14 \pm 0.71$ & $4.08 \pm 1.31$ & $<0.001$ \\
\hline Subcutaneous fat (cm) & $1.03 \pm 0.51$ & $3.49 \pm 0.98$ & $<0.001$ \\
\hline $\mathrm{SAP}(\mathrm{mmHg})$ & $107.4 \pm 11.6$ & $113.7 \pm 10.2$ & 0.003 \\
\hline $\mathrm{DAP}(\mathrm{mmHg})$ & $63.8 \pm 6.6$ & $66.5 \pm 6.3$ & 0.032 \\
\hline SAP (percentile) & $43.9 \pm 26.9$ & $62.6 \pm 29.2$ & $<0.001$ \\
\hline DAP (percentile) & $48.5 \pm 19.3$ & $58.2 \pm 17.3$ & 0.006 \\
\hline $\mathrm{HR}(\mathrm{b} / \mathrm{min})$ & $71.8 \pm 9.5$ & $70.0 \pm 9.9$ & 0.324 \\
\hline LV mass (g) & $119.4 \pm 43.0$ & $122.9 \pm 36.0$ & 0.716 \\
\hline RHI (-) & $1.86 \pm 0.48$ & $1.59 \pm 0.44$ & 0.037 \\
\hline Total cholesterol (mg/dL) & $130.5 \pm 39.7$ & $165.8 \pm 35.9$ & 0.001 \\
\hline LDL cholesterol (mg/dL) & $73.4 \pm 32.8$ & $100.4 \pm 30.8$ & 0.003 \\
\hline HDL cholesterol (mg/dL) & $46.8 \pm 12.5$ & $47.3 \pm 12.2$ & 0.868 \\
\hline Triglycerides (mg/dL) & $51.5 \pm 20.0$ & $89.8 \pm 68.0$ & 0.030 \\
\hline TryGI $(\mathrm{mg} / \mathrm{dL})^{2}$ & $7.62 \pm 0.44$ & $7.96 \pm 0.58$ & 0.145 \\
\hline Fasting glucose (mg/dL) & $78.5 \pm 7.7$ & $79.0 \pm 11.0$ & 0.935 \\
\hline Insulin (pmol/1) & $48.0 \pm 18.4$ & $128.7 \pm 83.6$ & $<0.001$ \\
\hline $\mathrm{HbA1c}(\mathrm{mmol} / \mathrm{mol})$ & $5.16 \pm 0.28$ & $5.43 \pm 0.31$ & 0.001 \\
\hline HOMA-iR & $0.89 \pm 0.31$ & $2.25 \pm 1.41$ & $<0.001$ \\
\hline
\end{tabular}

Data are presented as means \pm SD. Abbreviations: $\mathrm{BMI}=$ body mass index; $\mathrm{WHtR}=$ waist $/$ height ratio; $\mathrm{SAP}=$ systolic arterial pressure, $\mathrm{DAP}=$ diastolic arterial pressure, $\mathrm{HR}=$ heart rate, $\mathrm{LV}=$ left ventricle; $\mathrm{RHI}=$ reactive hyperemia index; $\mathrm{LDL}=$ low density lipoproteins; $\mathrm{HDL}=$ high density lipoproteins; TryGI = triglyceride-glucose index; $\mathrm{HbA} 1 \mathrm{c}=$ glycated haemoglobin; HOMA-IR = homeostatic model assessment for insulin resistance.

Resting values of key autonomic indices are presented in Table 2, which shows substantial equivalence of data. Conversely, SAP mean and (borderline) SAP variance are greater in the NW/OB groups.

Table 2. Resting values of key autonomic indices from RR interval variability in normal weight (NW) and overweight/obese children (OW/OB).

\begin{tabular}{|c|c|c|c|}
\hline Variables & NW & OW/OB & Significance \\
\hline Alpha index (ms/mmHg) & $28.0 \pm 19.0$ & $24.2 \pm 13.2$ & 0.203 \\
\hline RR interval (ms) & $849.9 \pm 118.8$ & $874.2 \pm 125.8$ & 0.304 \\
\hline RR variance $\left(\mathrm{ms}^{2}\right)$ & $4806.2 \pm 5381.2$ & $5894.5 \pm 6311.5$ & 0.340 \\
\hline $\mathrm{RR} L F$ a $\left(\mathrm{ms}^{2} / \mathrm{Hz}\right)$ & $1199.1 \pm 1372.7$ & $2423.9 \pm 3635.9$ & 0.166 \\
\hline $\mathrm{RR} H F$ a $\left(\mathrm{ms}^{2} / \mathrm{Hz}\right)$ & $2365.3 \pm 3824.9$ & $2423.9 \pm 3635.9$ & 0.934 \\
\hline RR LF nu (-) & $39.8 \pm 20.4$ & $42.0 \pm 17.9$ & 0.534 \\
\hline RR HF nu (-) & $53.0 \pm 21.1$ & $48.6 \pm 18.3$ & 0.235 \\
\hline RR LF/HF (-) & $1.27 \pm 1.75$ & $1.28 \pm 1.52$ & 0.976 \\
\hline RR LF f (Hz) & $0.10 \pm 0.03$ & $0.10 \pm 0.02$ & 0.374 \\
\hline RR HF f (Hz) & $0.30 \pm 0.07$ & $0.32 \pm 0.06$ & 0.113 \\
\hline$p \_0 \mathrm{v}(-)$ & $15.6 \pm 9.9$ & $17.4 \pm 12.6$ & 0.409 \\
\hline Resp HF f (Hz) & $0.31 \pm 0.06$ & $0.33 \pm 0.06$ & 0.139 \\
\hline SAP Mean (mmHg) & $106.1 \pm 12.3$ & $113.6 \pm 11.1$ & 0.001 \\
\hline SAP total power $\left(\mathrm{mmHg}^{2}\right)$ & $24.2 \pm 18.9$ & $32.1 \pm 25.2$ & 0.059 \\
\hline
\end{tabular}

Abbreviations; RR = interval between successive R waves; LF = low frequency; a = raw value; $\mathrm{HF}=$ high frequency;

$\mathrm{f}=$ frequency; $\mathrm{Hz}=$ Hertz; $p=$ beat sequence; Resp = respiration; $\mathrm{SAP}=$ systolic arterial pressure. 
Figure 1 reports the correlation matrix between major autonomic and metabolic/hemodynamic variables. Not surprisingly, yet interestingly, there is a clustered significant correlation between homogeneous indices. More specifically, autonomic variables show, as expected, strong intergroup correlations: e.g., alpha index vs. $R R$ variance $(r=0.602)$, $R R$ LF a $(r=0.454)$ or vs. $R R(r=0.547)$. Notably alpha index, a marker of overall gain of cardiac baroreflex, is also significantly correlated with TG ( $r=-0.261)$ and endothelial indices (RHI, $r=0.276)$.

Systolic arterial pressure indices correlate with left ventricular mass, TG (and TryG), insulin and HOMA, TMI, and with WHtR and BMI z-score.

A strong intragroup correlation characterizes clustering of metabolic indicators vs. visceral/subcutaneous fat.

Simple correlations, however, are very sensitive to outliers and cannot delve into the value of congruent correlations. Moreover, collinearity may be a problem. Given the complexity of the "organization of the whole mechanism of control" that we seek to explore, we need to choose "the appropriate technique of investigation" [33], and it is unlikely that simple deterministic models might be appropriate [34]. Accordingly, the bioengineering efforts to provide new "gold standard" indices [35] may have limited clinical relevance.

Conversely, given the practical goals of physiopathological research, it might be useful to consider that a limited number of hidden domains are responsible for the continuous transformation of multiple neurovisceral control functions into the complexity of a human organism [33]. Regarding beat-by-beat peripheral cardiovascular variabilities, prior studies with sympathetic nerve recordings provided evidence of "common central mechanisms governing sympathetic and parasympathetic rhythmic activity" [36]. Therefore, in recent studies we employed modern multivariate statistics to explore the possibility of reducing the large number of individual empirical indices into a smaller set of congruent variables, carrying specific aspects of relevant information. Specifically, in obese adults we observed that EFA suggested four domains (amplitude, frequency, pulse and pressure) [19].

The same approach was employed in the present study.

As shown in Figure 2, applying EFA to multidimensional data allowed us to reduce the number of variables describing the complex relationship between autonomic cardiac regulation and obesity. From an initial set of 27 explicit variables, accounting for $72.3 \%$ of data variance, EFA extracts 7 hidden components that more synthetically describe the reciprocal interrelations between ANS regulation and metabolic indicators. 


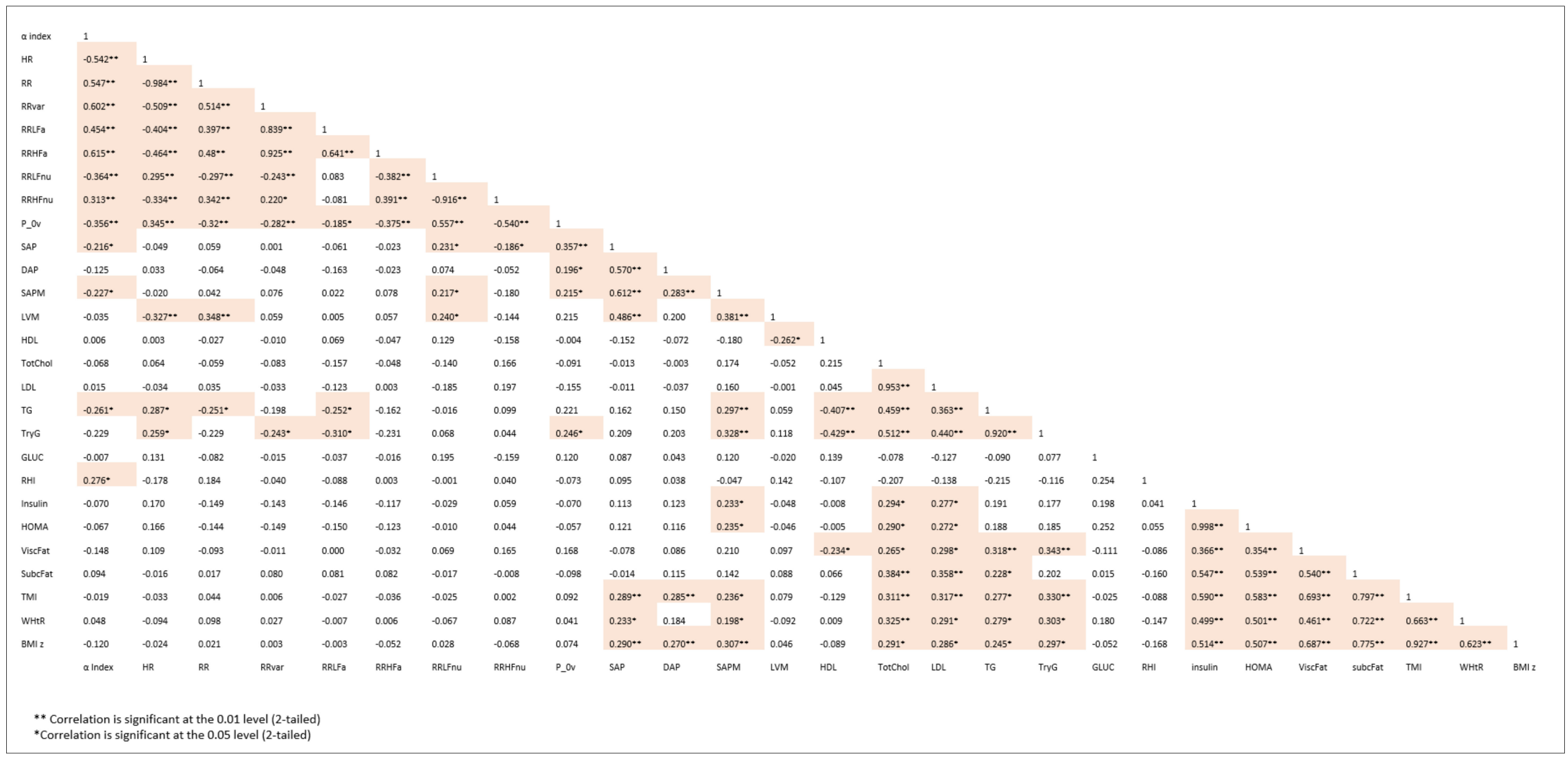

Figure 1. Correlation matrix between major autonomic and metabolic/hemodynamic variables. 


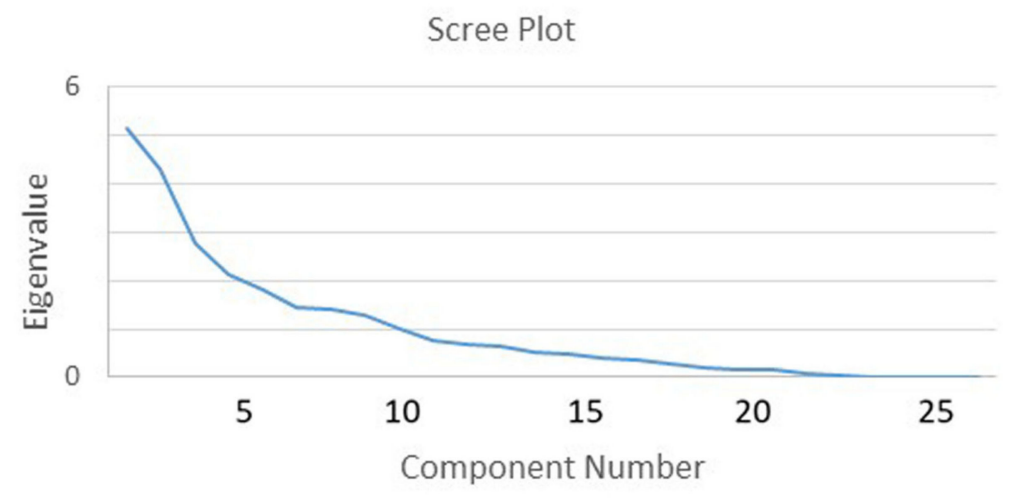

\begin{tabular}{|c|c|c|c|c|c|c|c|}
\hline \multirow[b]{3}{*}{$\%$} & & & $\begin{array}{r}\text { ted } \mathrm{Co} \\
\text { riance at } \\
\mathrm{C}\end{array}$ & $\begin{array}{l}\text { ponen } \\
\text { buted fo } \\
\text { ponent }\end{array}$ & $\begin{array}{l}\text { Matrix } \\
72.3 \%\end{array}$ & & \\
\hline & 1 & 2 & 3 & 4 & 5 & 6 & 7 \\
\hline & 20.200 & 16.800 & 10.400 & 8.100 & 6.700 & 5.500 & 5.300 \\
\hline AlpM & -0.012 & 0.697 & -0.323 & -0.135 & -0.023 & -0.063 & 0.150 \\
\hline HR & 0.044 & -0.651 & 0.423 & -0.325 & -0.001 & 0.228 & 0.077 \\
\hline RR & -0.042 & 0.660 & -0.419 & 0.333 & 0.005 & -0.196 & -0.055 \\
\hline RRTP & 0.021 & 0.938 & -0.044 & -0.010 & -0.036 & 0.003 & -0.036 \\
\hline RRLa & 0.019 & 0.869 & 0.259 & -0.114 & -0.040 & -0.032 & -0.055 \\
\hline RRHa & -0.003 & 0.852 & -0.216 & -0.020 & -0.015 & 0.037 & 0.018 \\
\hline RRLn & -0.032 & -0.133 & 0.895 & 0.184 & -0.037 & -0.078 & 0.033 \\
\hline RRHn & -0.009 & 0.130 & -0.907 & -0.101 & 0.079 & 0.113 & 0.028 \\
\hline P_Ov & -0.022 & -0.300 & 0.608 & 0.325 & -0.088 & 0.137 & -0.063 \\
\hline SBP & 0.115 & -0.065 & 0.146 & 0.853 & -0.008 & 0.018 & 0.058 \\
\hline DBP & 0.193 & -0.166 & -0.027 & 0.604 & -0.070 & 0.034 & 0.032 \\
\hline SAPM & 0.177 & 0.066 & 0.237 & 0.633 & 0.157 & 0.146 & 0.053 \\
\hline LVM & -0.063 & 0.163 & 0.101 & 0.604 & -0.016 & 0.124 & 0.050 \\
\hline coltot & 0.198 & -0.055 & -0.065 & -0.008 & 0.953 & 0.086 & -0.107 \\
\hline LDL & 0.194 & 0.005 & -0.122 & 0.012 & 0.890 & 0.107 & -0.094 \\
\hline $\mathrm{HDL}$ & -0.027 & -0.042 & 0.147 & -0.143 & 0.320 & -0.791 & -0.022 \\
\hline TG & 0.131 & -0.156 & 0.019 & 0.098 & 0.392 & 0.801 & -0.110 \\
\hline TryG & 0.113 & -0.180 & 0.038 & 0.182 & 0.427 & 0.776 & 0.003 \\
\hline GLUC & -0.229 & -0.011 & 0.098 & 0.037 & -0.077 & -0.044 & 0.690 \\
\hline $\mathrm{RHI}$ & -0.099 & 0.039 & -0.137 & 0.171 & -0.186 & -0.036 & 0.635 \\
\hline ins & 0.726 & -0.091 & 0.006 & -0.091 & 0.234 & 0.047 & 0.498 \\
\hline Visc & 0.620 & 0.037 & 0.177 & -0.069 & 0.072 & 0.354 & -0.163 \\
\hline Subc & 0.788 & 0.103 & 0.032 & -0.056 & 0.233 & 0.018 & -0.076 \\
\hline HOM & 0.717 & -0.090 & 0.017 & -0.083 & 0.236 & 0.045 & 0.524 \\
\hline TMI & 0.881 & -0.022 & -0.051 & 0.253 & 0.021 & 0.066 & -0.143 \\
\hline CValt & 0.637 & -0.013 & -0.147 & 0.244 & 0.010 & -0.049 & -0.145 \\
\hline bmiz & 0.859 & -0.029 & 0.016 & 0.254 & 0.010 & 0.032 & -0.229 \\
\hline
\end{tabular}

Figure 2. Exploratory factor analysis (EFA) of autonomic and metabolic indices from controls and patients. The seven hidden components that synthetize the reciprocal interrelations between autonomic nervous system regulation and metabolic indicators are reported in a different color.

In detail, employing the terminology that we described with this approach (see Methods), factor 1 (about $20 \%$ of total variance) links to the metabolic domain for the positive correlations with insulin and HOMA, visceral and subcutaneous fat, and TMI, WHtR, and BMI z-score; factor 2 (about $16 \%$ of total variance) relates to the amplitude and pulse domains, because it correlates positively with the alpha index, $R R, R R$ variance, $L F$ absolute and HF absolute, and negatively to HR. Factor 3 (about $10 \%$ of total variance) links to purely numerical indices of the oscillatory domain (RR LF nu, HF nu and P_0v). Factor 4 (about $8 \%$ of total variance) links to the pressure domain (SAP, DAP, SAP Mean). 
The remaining 5, 6 and 7 factors correspond, respectively, to lipids (cholesterol and TG), fasting glucose and endothelium performance. In brief, EFA provides a means to focus on the essential elements of individual factors considering the variable with the highest loading as representative of the information carried by the component. It should be added that for every factor it is possible to observe which empirical variable furnishes the greatest part of information. For instance, in the metabolic cluster (factor 1) the variables with highest loading (TMI and BMI z-score) should be confronted with visceral fat and HOMA that show the lowest loading. This suggests a relatively large variation in the link between empirical data and computed hidden factors. Likewise, for the HRV-derived indices we see factor 2 characterized by amplitude information (such as RR var), while factor 3 provides information on dimensionless (i.e., pure numbers) information. This shows, once more, that normalized variables carry a different meaning from absolute ones [37]. This finding, overall, suggests that autonomic nervous system control represents a pivotal mechanism that is secondary, as is obvious, only to insulin resistance and metabolic indices to describe the effect of obesity in the considered population. The autonomic derangement is more evident on the vascular end.

\section{Discussion}

The rising prevalence of obesity in the young population renders, nowadays, this condition not only a risk factor for many illnesses, particularly diabetes and cardiovascular diseases, but also a social emergency [38]. Accordingly, addressing obesity directly, particularly in young people, has become a must in preventive strategy, where lifestyle changes outperform the usual pharmacological tools considering the young age [39]. As a corollary, advanced, practical knowledge of underlying physiopathological mechanisms, like ANS control, which may be improved by behavioral interventions $[14,15,40]$ such as diet and exercise, becomes mandatory.

This observational study confirms and extends previous observations of ours indicating a generalized involvement of cardiac and arterial systems in youth obesity [41]. In particular, an increase in LV dimensions and mass as well as in common carotid diameter and distension were found, mainly reflecting adaptation to a body-size-induced increase in hemodynamic load, and an increase in carotid intima-media thickness IMT related to both adaptive remodeling and metabolic risk.

Here, we now report a significant correlation between metabolic indices (as triglycerides), endothelial control (as RHI) and autonomic control, particularly in its vascular end (alpha index), which may play a mechanistic role in the above reported changes.

To interpret the alterations of multiple control systems, as in the present study, it is important to recognize that neural visceral regulation is not simply based on purely efferent, antagonistic, sympathetic and parasympathetic innervation (described approximately by a dynamic balance), but beat-by-beat regulation is the result of a complex hierarchical multilevel system [42,43], comprising positive-negative feedback, pointing at a unitary functional aim [33,44]. Given this complexity, simple statistics, like correlation, can suggest functional links, but correlation is very sensitive to computational drawbacks, like collinearity. Moreover, it is difficult to provide a clear-cut interpretation of underlying mechanisms. Indeed, even now, HRV derived indices do not have an agreed physiopathological meaning. This applies in particular considering the different way of computing variables (according to a novel systematization of metrics) [37]. According to this view (applicable to other cardiac properties, like ventricular performance), physical indices (having a measurable metric, e.g., heart volume) and numerical quantities (e.g., EF that is dimensionless), obtained with a division (ratio), provide different, possibly complementary, information. In the case of HRV we may consider e.g., RR interval or variance (units are $\mathrm{msec}$ or $\mathrm{msec}^{2}$ ) or the normalized LF and HF components, or LF/HF ratio (dimensionless). We reported several years ago that nu variables are more efficient than $R R$ variance in detecting conditions characterized by increased sympathetic activity (as with standing up) [45]. In the present study we report for completeness a table of simple correlations, merely to point out the 
clustering of congruent variables, and the relatively weak link between variables pertaining to different domains. To make this point, consider the correlation between alpha index (a measure of the cardiac baroreflex) and RHI (a measure of endothelial functionality): the obtained $\mathrm{r}$ value of 0.276 might seem small. It is, however, of the same order of magnitude $(r=0.278)$ as the correlation between arterial pressure and alpha index in humans [46], suggesting that relationships between physiopathological indices may be more complex than those dictated by simple algebra.

A more rewarding way of extracting coded information from cardiovascular variables might be provided by multisystem analysis or multivariate statistics.

We have been exploring this possibility using advanced multifactorial statistical analysis (as exploratory factor analysis, EFA) applied to heart rate variability. We have recently shown that EFA $[19,20]$, while reducing the large number of autonomic indices provided by usual techniques, also extracts a smaller number of hidden variables that carry a functional, integrated, meaning. In the specific case of obesity, in adults, we found that EFA reduces the totality $(n=16)$ of indices obtained from HRV to only five domains, containing more than $80 \%$ of the embedded information. According to this approach, the following are the critical elements of autonomic cardiovascular control playing a role in obesity dysautonomia: strong role for pulse and pressure; almost strong for alpha index, weak role for amplitude and oscillations [19]. It may be added that for these types of data the comparison between groups is better served by considering both differences in position and in distribution. This last aspect may even be prevalent, although it is rather complex to compute, and requires larger study populations.

Although we cannot directly transpose the adult approach to these data from children, we may also confirm that in the present young population pressure and pulse seem to relate strongly to the various metabolic elements of childhood obesity. Moreover, preliminary distribution analysis suggests that, at this young age, metabolism and pressure (which is most sensitive in adults) [19] values are significantly different between normal weight and children with obesity, while cardiac autonomic indices are not (data not presented for simplicity). Thus, we may hypothesize that in childhood obesity the initial stage is characterized by hemodynamic alterations (pressure and endothelial function), but not yet by autonomic cardiac impairment. This hypothesis renders more cogent the introduction of lifestyle changes to reverse the gradual autonomic dysfunction that is present in obese or metabolically unsound individuals [47].

Triglycerides, insulin resistance as per HOMA, and BMI appear linked to arterial pressure indices (Figure 2). Systemic pressure is also, as expected, correlated to LVM. Amplitude and oscillation domains carry a weak relation with metabolic and anthropometric indices of childhood obesity. Conversely, the alpha index shows in children a stronger link with metabolic obesity (TG) and endothelial (RHI) indicators. This analysis might be useful to detect the initial ANS impairment in overweight/obese children, even if unpaired $t$-test analysis, considering the two small groups of this study, fails in identifying significant differences in ANS indices. Indeed, cardiac dysautonomia may take a long time to become manifest [47].

Obesity has a negative, progressive, impact on health, being a risk for many diseases, including cardiovascular disorders. In adults, established obesity is characterized by increased sympathetic activity, with a relative relation with severity of the condition, often associated to arterial hypertension.

Here, we analyzed autonomic cardiovascular control in children with overweight and obesity, showing a substantial equivalence of resting values of key autonomic indices in normal weight and overweight/obesity. Since we also found a clear link between autonomic variables and metabolic indicators, as well as with left ventricle mass, our results support the contention that subtle changes in autonomic nervous system control could be considered a possible way to chase early preclinical cardiovascular derangement in the pediatric obese population, even before the occurrence of frank autonomic dysregulation. 
This finding should be interpreted considering that autonomic nervous system imbalance is a potent risk factor for adverse cardiovascular events [11-13]. Nevertheless, only a limited number of studies have analyzed the autonomic function in obese pediatric populations [11-14,48], and an autonomic dysfunction has been reported, as a result of sympathovagal imbalance. In our patients, blood pressure was higher in the overweight/obese group compared to normal weight children, in the absence of substantial differences in autonomic indices. These data suggest the testable hypothesis that a mild increase in blood pressure may be considered an early sign of cardiovascular dysregulation, expressing early derangement of vascular health (as suggested by the altered RHI). The occurrence of endothelial dysfunction before the appearance of structural arterial damage and autonomic impairment fits well with Joyner's [40] "risk factor gap", as a strong support for exercise and diet as early preventive treatments. Obesity-related hypertension is associated with increased cardiovascular morbidity and mortality, and early diagnosis and treatment for blood pressure control and weight reduction is essential [49].

Hypertension often coexists with IR [50]. IR may contribute to increased blood pressure through several mechanisms, including the enhanced tissue angiotensin II and aldosterone activities, increased sympathetic nervous system activity and oxidative stress; the genetic predisposition for IR and hypertension could be also considered [51]. The links between autonomic indices and insulin levels and HOMA-IR could confirm the role of hyperinsulinemia in the sympathetic and vagal activity leading to hypertension [52]. In our young population we may only witness the initial derangement of cardiovascular regulation, which may progress from arterial dysregulation to increased arterial pressure, and subsequently comprise altered cardiac autonomic regulation. In our study, the link between visceral and subcutaneous fat and adiposity indices and major autonomic variables confirmed that obesity itself leads to cardiovascular alterations [19]. Adipose tissue is an endocrine organ modulating an inflammatory state and metabolic processes leading to consequences of the cardiovascular system [53] that we have not addressed here.

ANS impairment characterizes many chronic diseases in adulthood, such as diabetes, arterial hypertension and coronary artery disease [30,46], being one of the most important targets of preventive strategies $[39,54,55]$. Overweight and obesity in children, although negative as a clinical risk factor, represent, however, conditions that are easy to manage by employing behavioral lifestyle interventions. These strategies, further to other metabolic, immunological and hemodynamic benefits, are capable of improving autonomic nervous system impairment $[14,15,40]$.

A few study limitations should be acknowledged, starting from the relatively small sample size that can limit power analysis. Therefore, further large population studies are mandatory. Additionally, this is a retrospective study and results may be affected by some bias. Finally, no family history on cardiovascular diseases and/or other potential cardiovascular risk factors, such as nutritional behavior, physical activity level and prenatal environment were analyzed.

\section{Conclusions}

Pediatric patients with obesity show a link between indices of autonomic and endothelial function, fat distribution and metabolic profile. A greater blood pressure value at rest in control compared to overweight/obese groups was detected in the presence of substantial equivalence of resting values of autonomic indices. Even though the strategies to reduce cardiovascular risk have not yet been fully elucidated, the role of autonomic control optimization to prevent, or at least delay, further deterioration and the occurrence of structural vascular damage, should be not excluded. This multidimensional analysis might help to better characterize the individual progression of the various aspects of altered metabolic, hemodynamic and cardiac autonomic regulation. 
Author Contributions: Conceptualization, V.C. and D.L.; methodology, D.L., M.M., M.P., G.F., M.K.; formal analysis, D.L., M.M., C.P.; investigation, D.L., M.M., C.P., G.F., M.K.; resources, V.C., D.L., G.Z.; data curation, D.L., C.P.; writing—original draft preparation, D.L., M.P., V.C., C.P., G.F.; writingreview and editing, D.L., M.P., V.C., C.P., G.Z.; funding acquisition, V.C., C.P. All authors have read and agreed to the published version of the manuscript.

Funding: This study was in part funded by the Regione Toscana (Tuscany Region) Project Code B55E09000560002.

Institutional Review Board Statement: The protocol of the study followed the principles of the Declaration of Helsinki and Title 45, US Code of Federal Regulations, Part 46, Protection of Human Subjects (revised 13 November 2001, effective 13 December 2001) and was regularly approved by the institutional Ethics Committee of the Area Vasta Nord-Ovest (CEAVNO).

Informed Consent Statement: Children's guardians gave their written consent to participate; consent from the children and adolescents was also obtained.

Data Availability Statement: The data presented in this study are available on request from the corresponding author.

Conflicts of Interest: The authors declare no conflict of interest.

\section{References}

1. World Health Organization. Noncommunicable diseases: Childhood overweight and obesity. Available online: https://www. who.int/dietphysicalactivity/childhood/en/ (accessed on 28 July 2021).

2. NCD Risk Factor Collaboration (NCD-RisC). Worldwide trends in body-mass index, underweight, overweight, and obesity from 1975 to 2016: A pooled analysis of 2416 population-based measurement studies in 128.9 million children, adolescents, and adults. Lancet 2017, 390, 2627-2642. [CrossRef]

3. Lauria, L.; Spinelli, A.; Buoncristiano, M.; Nardone, P. Decline of childhood overweight and obesity in Italy from 2008 to 2016 : Results from 5 rounds of the population-based surveillance system. BMC Public Health 2019, 19, 618. [CrossRef]

4. Després, J.P. Body fat distribution and risk of cardiovascular disease: An update. Circulation 2012, 126, 1301-1313. [CrossRef]

5. Vizzuso, S.; Del Torto, A.; Dilillo, D.; Calcaterra, V.; Di Profio, E.; Leone, A.; Gilardini, L.; Bertoli, S.; Battezzati, A.; Zuccotti, G.V.; et al. Visceral Adiposity Index (VAI) in Children and Adolescents with Obesity: No Association with Daily Energy Intake but Promising Tool to Identify Metabolic Syndrome (MetS). Nutrients 2021, 13, 413. [CrossRef]

6. Leone, A.; Vizzuso, S.; Brambilla, P.; Mameli, C.; Ravella, S.; De Amicis, R.; Battezzati, A.; Zuccotti, G.; Bertoli, S.; Verduci, E. Evaluation of Different Adiposity Indices and Association with Metabolic Syndrome Risk in Obese Children: Is there a Winner? Int. J. Mol. Sci. 2020, 21, 4083. [CrossRef] [PubMed]

7. Malavazos, A.E.; Capitanio, G.; Milani, V.; Ambrogi, F.M.; Matelloni, I.A.; Basilico, S.; Dubini, C.; Sironi, F.M.; Stella, E.; Castaldi, S.; et al. Tri-Ponderal Mass Index vs body Mass Index in discriminating central obesity and hypertension in adolescents with overweight. Nutr. Metab. Cardiovasc. Dis. 2021, 31, 1613-1621. [CrossRef] [PubMed]

8. Licenziati, M.R.; Iannuzzo, G.; Morlino, D.; Campana, G.; Renis, M.; Iannuzzi, A.; Valerio, G. Fat mass and vascular health in overweight/obese children. Nutr. Metab. Cardiovasc. Dis. 2021, 31, 1317-1323. [CrossRef] [PubMed]

9. Angoorani, H.; Karimi, Z.; Naderi, F.; Mazaherinezhad, A. Is ultrasound-measured abdominal fat thickness a reliable method for predicting metabolic diseases in obese and overweight women? Med. J. Islam. Repub. Iran. 2018, 28, 32-78. [CrossRef]

10. Liao, D.; Rodríguez-Colón, S.M.; He, F.; Bixler, E.O. Childhood obesity and autonomic dysfunction: Risk for cardiac morbidity and mortality. Curr. Treat. Options Cardiovasc. Med. 2014, 16, 342. [CrossRef] [PubMed]

11. Lucini, D.; de Giacomi, G.; Tosi, F.; Malacarne, M.; Respizzi, S.; Pagani, M. Altered cardiovascular autonomic regulation in overweight children engaged in regular physical activity. Heart 2013, 99, 376-381. [CrossRef]

12. Calcaterra, V.; Vandoni, M.; Correale, L.; Larizza, D.; DeBarbieri, G.; Albertini, R.; Tinelli, C.; Arpesella, M.; Bernardi, L. Deep breathing acutely improves arterial dysfunction in obese children: Evidence of functional impairment? Nutr. Metab. Cardiovasc. Dis. 2014, 24, 1301-1309. [CrossRef]

13. Calcaterra, V.; Vandoni, M.; Debarbieri, G.; Larizza, D.; Albertini, R.; Arpesella, M.; Bernardi, L. Deep breathing improves blunted baroreflex sensitivity in obese children and adolescents with insulin resistance. Int. J. Cardiol. 2012, 168, 1614-1615. [CrossRef]

14. Lucini, D.; Zuccotti, G.V.; Scaramuzza, A.; Malacarne, M.; Gervasi, F.; Pagani, M. Exercise might improve cardiovascular autonomic regulation in adolescents with type 1 diabetes. Acta Diabetol. 2013, 50, 341-349. [CrossRef]

15. Lucini, D.; Malacarne, M.; Gatzemeier, W.; Pagani, M. A simple home-based lifestyle intervention program to improve cardiac autonomic regulation in patients with increased cardiometabolic risk. Sustainability 2020, 12, 7671. [CrossRef]

16. Brasil, I.; Monteiro, W.; Lima, T.; Seabra, A.; Farinatti, P. Effects of judo training upon body composition, autonomic function, and cardiorespiratory fitness in overweight or obese children aged 8-13 years. J. Sports Sci. 2020, 38, 2508-2516. [CrossRef]

17. Speer, K.E.; Naumovski, N.; Semple, S.; McKune, A.J. Lifestyle Modification for Enhancing Autonomic Cardiac Regulation in Children: The Role of Exercise. Children 2019, 6, 127. [CrossRef] [PubMed] 
18. Santana, M.; Kliszczewicz, B.; Vanderlei, F.M.; Monteiro, L.; Martiniano, E.C.; de Moraes, Y.M.; Mangueira, L.B.; Alcantara, G.C.; da Silva, J.; Benjamim, C.; et al. Autonomic responses induced by aerobic submaximal exercise in obese and overweight adolescents. Cardiol. Young 2019, 29, 169-173. [CrossRef]

19. Solaro, N.; Pagani, M.; Lucini, D. Altered Cardiac Autonomic Regulation in Overweight and Obese Subjects: The Role of Age-and-Gender-Adjusted Statistical Indicators of Heart Rate Variability and Cardiac Baroreflex. Front. Physiol. 2021, 11, 567312. [CrossRef]

20. Lucini, D.; Solaro, N.; Pagani, M. Autonomic Differentiation Map: A Novel Statistical Tool for Interpretation of Heart Rate Variability. Front. Physiol. 2018, 9, 401. [CrossRef] [PubMed]

21. Calcaterra, V.; De Giuseppe, R.; Biino, G.; Mantelli, M.; Marchini, S.; Bendotti, G.; Madè, A.; Avanzini, M.A.; Montalbano, C.; Cossellu, G.; et al. Relation between circulating oxidized-LDL and metabolic syndrome in children with obesity: The role of hypertriglyceridemic waist phenotype. J. Pediatric Endocrinol. Metab. 2017, 30, 1257-1263. [CrossRef]

22. World Health Organization. BMI-for-age (5-19 years). Available online: https:/ /www.who.int/tools/growth-reference-data-for5to19-years/indicators/bmi-for-age (accessed on 28 July 2021).

23. Mameli, C.; Krakauer, N.Y.; Krakauer, J.C.; Bosetti, A.; Ferrari, C.M.; Moiana, N.; Schneider, L.; Borsani, B.; Genoni, T.; Zuccotti, G. The association between a body shape index and cardiovascular risk in overweight and obese children and adolescents. PLoS ONE 2018, 13, e0190426. [CrossRef]

24. Hudda, M.T.; Fewtrell, M.S.; Haroun, D.; Lum, S.; Williams, J.E.; Wells, J.; Riley, R.D.; Owen, C.G.; Cook, D.G.; Rudnicka, A.R.; et al. Development and validation of a prediction model for fat mass in children and adolescents: Metaanalysis using individual participant data. BMJ 2019, 366, 14293. [CrossRef]

25. Del Ry, S.; Cabiati, M.; Bianchi, V.; Caponi, L.; Maltinti, M.; Caselli, C.; Kozakova, M.; Palombo, C.; Morizzo, C.; Marchetti, S.; et al. C-type natriuretic peptide is closely associated to obesity in Caucasian adolescents. Clin. Chim. Acta Int. J. Clin. Chem. 2016, 460, 172-177. [CrossRef]

26. Matthews, D.R.; Hosker, J.P.; Rudenski, A.S.; Naylor, B.A.; Treacher, D.F.; Turner, R.C. Homeostasis model assessment: Insulin resistance and $\beta$-cell function from fasting plasma glucose and insulin concentrations in man. Diabetologia 1985, 28, 412-419. [CrossRef] [PubMed]

27. Simental-Mendía, L.E.; Rodríguez-Morán, M.; Guerrero-Romero, F. The Product of Fasting Glucose and Triglycerides as Surrogate for Identifying Insulin Resistance in Apparently Healthy Subjects. Metab. Syndr. Relat. Disord. 2008, 6, 299-304. [CrossRef] [PubMed]

28. Haller, M.J.; Stein, J.; Shuster, J.; Theriaque, D.; Silverstein, J.; Schatz, D.A.; Earing, M.G.; Lerman, A.; Mahmud, F.H. Peripheral artery tonometry demonstrates altered endothelial function in children with type 1 diabetes. Pediatr Diabetes 2007, 8, 193-198. [CrossRef] [PubMed]

29. Palombo, C.; Kozakova, M.; Morizzo, C.; Gnesi, L.; Barsotti, M.C.; Spontoni, P.; Massart, F.; Salvi, P.; Balbarini, A.; Saggese, G.; et al. Circulating endothelial progenitor cells and large artery structure and function in young subjects with uncomplicated type 1 diabetes. Cardiovasc. Diabetol. 2011, 10, 88. [CrossRef]

30. Sabir, N.; Pakdemirli, E.; Sermez, Y.; Zencir, M.; Kazil, S. Sonographic assessment of changes in thickness of different abdominal fat layers in response to diet in obese women. J. Clin. Ultrasound JCU 2013, 31, 26-30. [CrossRef] [PubMed]

31. Ribeiro-Filho, F.F.; Faria, A.N.; Kohlmann, O., Jr.; Ajzen, S.; Ribeiro, A.B.; Zanella, M.T.; Ferreira, S.R. Ultrasonography for the evaluation of visceral fat and cardiovascular risk. Hypertension 2001, 38, 713-717. [CrossRef]

32. Pagani, M.; Somers, V.; Furlan, R.; Orto, S.D.; Conway, J.; Baselli, G.; Cerutti, S.; Sleight, P.; Malliani, A. Changes in Autonomic Regulation Induced by Physical Training in Mild Hypertension. Hypertension 1988, 12, 600-610. [CrossRef] [PubMed]

33. Hess, W.R. The Central Control of the Activity of Internal Organs. In Nobel Lectures, Physiology or Medicine 1942-1962; Elsevier: Amsterdam, The Netherlands, 1949; Available online: https://www.nobelprize.org/prizes/medicine/1949/hess/lecture/ (accessed on 28 July 2021).

34. Ahn, A.C.; Tewari, M.; Poon, C.S.; Phillips, R.S. The clinical applications of a systems approach. PLoS Med. 2006, 3, 0956-0960. [CrossRef] [PubMed]

35. Sassi, R.; Cerutti, S.; Lombardi, F.; Malik, M.; Huikuri, H.V.; Peng, C.K.; Schimdt, G.; Yamamoto, Y. Advances in heart rate variability signal analysis: Joint position statement by the e-Cardiology ESC Working Group and the European Heart Rhythm Association co-endorsed by the Asia Pacific Heart Rhythm Society. Europace 2015, 17, 1341-1353. [CrossRef] [PubMed]

36. Pagani, M.; Montano, N.; Porta, A.; Malliani, A.; Abboud, F.M.; Birkett, C.; Somers, V.K. Relationship between spectral components of cardiovascular variabilities and direct measures of muscle sympathetic nerve activity in humans. Circulation 1997, 95, 1441-1448. [CrossRef]

37. Kerkhof, P.L.M.; Peace, R.A.; Handly, N. Ratiology and a Complementary Class of Metrics for Cardiovascular Investigations. Physiology 2019, 34, 250-263. [CrossRef]

38. Allen, L.N.; Feigl, A.B. Reframing non-communicable diseases as socially transmitted conditions. Lancet. Glob. Health 2017, 5, e644-e646. [CrossRef]

39. Valerio, G.; Maffeis, C.; Saggese, G.; Ambruzzi, M.A.; Balsamo, A.; Bellone, S.; Bergamini, M.; Bernasconi, S.; Bona, G.; Calcaterra, V.; et al. Diagnosis, treatment and prevention of pediatric obesity: Consensus position statement of the Italian Society for Pediatric Endocrinology and Diabetology and the Italian Society of Pediatrics. Ital. J. Pediatrics 2018, 44, 88. [CrossRef] 
40. Michael, J.; Joyner, M.J.; Green, D.J. Exercise protects the cardiovascular system: Effects beyond traditional risk factors. J. Physiol. 2009, 587, 5551-5558.

41. Kozakova, M.; Morizzo, C.; Bianchi, V.; Marchetti, S.; Federico, G.; Palombo, C. Hemodynamic overload and intra-abdominal adiposity in obese children: Relationships with cardiovascular structure and function. Nutr. Metab. Cardiovasc. Dis. 2016, 26, 60-66. [CrossRef] [PubMed]

42. Malliani, A.; Pagani, M.; Lombardi, F.; Cerutti, S. Cardiovascular neural regulation explored in the frequency domain. Circulation 1991, 84, 482-492. [CrossRef] [PubMed]

43. Fukuda, K.; Kanazawa, H.; Aizawa, Y.; Ardell, J.L.; Shivkumar, K. Cardiac Innervation and Sudden Cardiac Death. Circ Res. 2015, 116, 2005-2019. [CrossRef]

44. Sun, M.K. Central neural organization and control of sympathetic nervous system in mammals. Prog. Neurobiol. 1995, 47, 157-233. [CrossRef]

45. Malliani, A.; Pagani, M.; Furlan, R.; Guzzetti, S.; Lucini, D.; Montano, N.; Cerutti, S.; Mela, G.S. Individual recognition by heart rate variability of two different autonomic profiles related to posture. Circulation 1997, 96, 4143-4145. [CrossRef]

46. Lucini, D.; Mela, G.S.; Malliani, A.; Pagani, M. Impairment in cardiac autonomic regulation preceding arterial hypertension in humans: Insights from spectral analysis of beat-by-beat cardiovascular variability. Circulation 2002, 106, 2673-2679. [CrossRef] [PubMed]

47. Lucini, D.; Cusumano, G.; Bellia, A.; Kozakova, M.; DiFede, G.; Lauro, R.; Linosa Study Group. Is reduced baroreflex gain a component of the metabolic syndrome? Insights from the LINOSA study. J. Hypertens. 2006, 24, 361-370. [CrossRef]

48. Lucini, D.; Zuccotti, G.; Malacarne, M.; Scaramuzza, A.; Riboni, S.; Palombo, C.; Pagani, M. Early progression of the autonomic dysfunction observed in pediatric type 1 diabetes mellitus. Hypertension 2009, 54, 987-994. [CrossRef]

49. Wühl, E. Hypertension in childhood obesity. Acta Paediatr. 2019, 108, 37-43. [CrossRef]

50. Zhou, M.S.; Wang, A.; Yu, H. Link between insulin resistance and hypertension: What is the evidence from evolutionary biology? Diabetol. Metab. Syndr. 2014, 6, 12. [CrossRef]

51. Mancusi, C.; Izzo, R.; di Gioia, G.; Losi, M.A.; Barbato, E.; Morisco, C. Insulin Resistance the Hinge Between Hypertension and Type 2 Diabetes. High Blood Press. Cardiovasc. Prev. 2020, 27, 515-526. [CrossRef]

52. Curtis, B.M.; O'Keefe, J.H. Autonomic tone as a cardiovascular risk factor: The dangers of chronic fight or flight. Mayo. Clin. Proc. 2002, 77, 45-54. [CrossRef] [PubMed]

53. Forkert, E.C.O.; Rendo-Urteaga, T.; Nascimento-Ferreira, M.V.; Ferreira de Moraes, A.C.; Moreno, L.A.; Barbosa de Carvalho, H. Abdominal obesity and cardiometabolic risk in children and adolescents, are we aware of their relevance? Nutrire 2016, 41, 15. [CrossRef]

54. James Fisher, J.P.; Fadel, P.J. Therapeutic strategies for targeting excessive central sympathetic activation in human hypertension. Exp. Physiol. 2010, 95, 572-580. [CrossRef] [PubMed]

55. Solaro, N.; Lucini, D.; Pagani, M. Handling missing data in observational clinical studies concerning cardiovascular risk: An insight into critical aspects. In Data Science-Innovative Developments in Data Analysis and Clustering; Series on Studies in Classification, Data Analysis, and Knowledge Organization; Palumbo, F., Montanari, A., Vichi, M., Eds.; Springer: Berlin/Heidelberg, Germany, 2017; pp. 131-144. ISBN 978-3-319-55722-9. 